\title{
Investigation of Image-based Lifetime PSP Measurements with Sinusoidal Excitation Light
}

\author{
Daisuke Yorita $^{1}$, Ulrich Henne ${ }^{2}$, Christian Klein ${ }^{3}$ \\ Institute of Aerodynamics and Flow Technology, German Aerospace Center (DLR), \\ D-37073 Göttingen, Germany \\ Mizue Munekata ${ }^{4}$ \\ Department of Mechanical and Mathematical Engineering, Kumamoto University, \\ 860-8555 Kumamoto, Japan \\ Gerhard A. Holst ${ }^{5}$ \\ PCO AG, D-93309 Kelheim, Germany
}

\begin{abstract}
The applicability of frequency-domain fluorescence lifetime imaging (FLIM) with a newly developed CMOS camera sensor (pco.flim) to Pressure-Sensitive Paint (PSP) measurements in wind tunnel tests was investigated. Two lifetime parameters, the phase angle and demodulation index, can be used as a function of pressure in FLIM technique. First, the characteristics of FLIM-PSP technique were examined in calibration tests. The two lifetime parameters, a phase shift and a modulation index, show different sensitivity to pressure and temperature. This indicates a potential of simultaneous pressure and temperature measurement of the FLIM-PSP technique. Next, the FLIM-PSP technique is demonstrated in a transonic wind tunnel using a delta-wing model. The obtained PSP results successfully visualize the complex pressure distribution on the model surface and the quantitative agreement with conventional pressure tap data is good.
\end{abstract}

\section{Introduction}

$\mathrm{P}$ ressure Sensitive Paint (PSP) measurement technique is based on the dependence of the intensity or decay time of its luminescence on pressure, brought about by oxygen quenching [1]. PSP is usually exited by light at an appropriate wavelength (e.g. UV-Light) and its pressure dependent luminescence intensity or lifetime is detected by a camera system (CCD or CMOS). In the method based on the luminescent lifetime, two basic types of measurement exist: The first type is the time-domain lifetime method, which is mostly used in various PSP applications. For this method a pulsed light is used to excite the paint and the pressure dependent time constant is determined from decay curve of luminescence intensity. The second type is the frequency-domain fluorescence lifetime imaging (FLIM) where modulated light is used to excite the paint and the PSP luminescence is simultaneously detected to calculate pressure dependent phase shift or amplitude. Only few applications were reported using this method [2].

Recently, a new CMOS image sensor has been developed by CSEM and PCO for frequency-domain FLIM system and equipped in the pco.flim camera for fluorescence lifetime imaging in microscopy [3]. In our previous work, the frequency-domain lifetime PSP technique (FLIM-PSP) with the pco.flim camera was investigated in calibration tests and a low-speed wind tunnel test [4]. The pressure distribution caused by the vortex on a delta wing was successfully visualized. However, the results were limited to qualitative analysis mainly due to instability of the modulation light source.

In this study, the FLIM-PSP technique is further investigated with pco.flim camera and more stable high power modulation light source. First, characteristics of the FLIM-PSP technique are investigated in calibration tests. Then, a transonic wind tunnel test is performed with the same system. The pressure distribution produced by the vortex on

\footnotetext{
${ }^{1}$ Research Scientist, Department Experimental Methods, daisuke.yorita@dlr.de, AIAA Member

${ }^{2}$ Research Scientist, Department Experimental Methods, christian.klein@dlr.de

${ }^{3}$ Research Scientist, Department Experimental Methods, ulrich.henne@dlr.de, AIAA Associate Fellow

${ }^{4}$ Associate Professor, munekata@gpo.kumamoto-u.ac.jp

${ }^{5}$ Research Engineer, gerhard.holst@pco.de
} 
the delta-wing model is visualized and results of the FLIM-PSP technique are compared with those of the conventional lifetime PSP technique.

\section{FLIM-PSP Technique}

\section{A. Principle}

Figure 1 shows a typical response of PSP luminescence to a modulated (sinusoidal) excitation light with a frequency $f_{\text {mod }}$. The luminescence phase and amplitude is dependent on the luminescence lifetime of PSP which is determined by the partial oxygen pressure i.e. local air pressure. Two parameters can be used as a function of pressure (and also temperature). The first parameter is the phase angle $\Phi$ or phase shift between excitation and emission from PSP, which is estimated as a time delay. Second parameter is the demodulation index of emission $m_{e m}$, which is the ratio of the amplitude $a_{e m}$ to the average intensity $b_{e m}$.

In the image-based technique, these quantities can be calculated from gated images obtained during the modulation period. Here four gated image method is introduced [3]. The gated images are acquired delayed corresponding to phase of $0,90,180$, and $270^{\circ}$ of the modulation signal with the width of each image being half of the modulation period. Each gated image is denoted as $I_{1}, I_{2}, I_{3}$ and $I_{4}$, respectively. Figure 2 shows the timing of the second gated image $I_{2}$ as an example. From these four gated images, the tangent of phase angle $\Phi$ and the demodulation index $m_{e m}$ can be calculated by Eq. 1 and Eq. 2 .

$$
\begin{gathered}
\tan \emptyset=\frac{I_{4}-I_{2}}{I_{1}-I_{3}} \\
m_{e m}=\frac{a_{e m}}{b_{e m}}=2 \cdot \frac{\sqrt{\left(I_{1}-I_{3}\right)^{2}+\left(I_{4}-I_{2}\right)^{2}}}{I_{1}+I_{2}+I_{3}+I_{4}}
\end{gathered}
$$

Usually, the PSP emission intensity for a single-shot gated image is very small. Therefore, a multi-exposure camera is required for the accumulation of PSP signal with thousands periods of modulation.

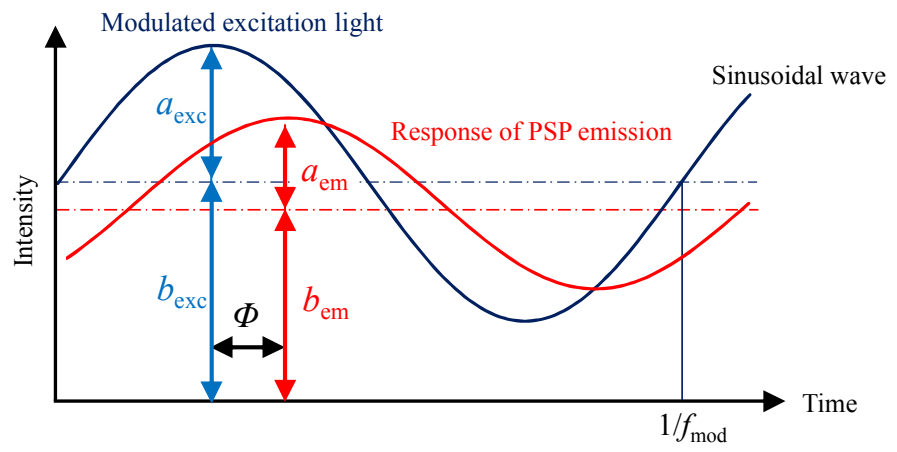

Figure 1. Typical response of PSP luminescence to a modulated (sinusoidal) excitation light.

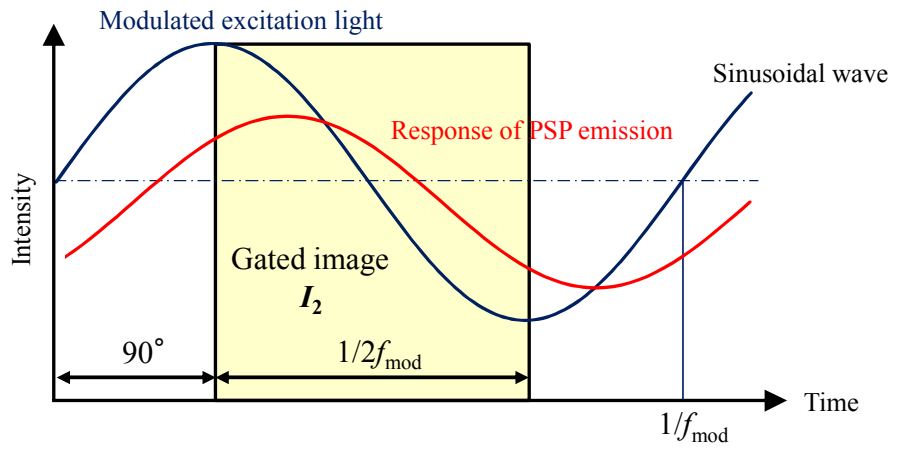

Figure 2. Timing of the second gated image $I_{2}$.

American Institute of Aeronautics and Astronautics 


\section{B. pco.flim camera}

The FLIM technique is applied to acquire pressure distribution from PSP using a pco.flim camera (PCO AG), which has an in-pixel dual tap control CMOS image sensor, $1008 \times 1008$ pixels resolution and 14 bit A/D converter [3]. The pco.flim camera is able to accumulate multi-exposure images using the following mechanism: The CMOS image sensor is composed of a single photodiode and two charge collection sites, called tap A and tap B as shown in Fig. 3. The sensor can selectively guide photo-generated charge carriers to each tap triggered by an external signal (tap control signal). When tap A is active, the charge carriers are guided and accumulated into tap A charge bucket. Then the control signal is switched, the carriers drift into the tap B bucket. By switching the control signal again, the accumulation of the carriers in tap A is restarted. In order to use this CMOS sensor for FLIM-PSP technique, following needs to be considered:

(1) Use of same frequency for the excitation light modulation and the tap control signal. The pco.flim camera is able to output the frequency master signal for the excitation light.

(2) The duty cycle of the tap control signal is set to $50 \%$. This means that the tap A and B have same integration time with half the period of the modulation.

(3) When the delay from the excitation light modulation to the tap control signal is zero, the tap A collects the charge carriers during first half of the light modulation and the tap B collects during second half of the light modulation. They are corresponding to the gated images of $I_{1}$ and $I_{3}$ as introduced in the section II.A.

(4) When the delay of a quarter of the period is given from the light modulation to the tap control signal, the images obtained by tap A and B correspond to $I_{2}$ and $I_{4}$ respectively.

As a result, a set of PSP images $\left(I_{1}, I_{3}\right)$ or $\left(I_{2}, I_{4}\right)$ can be obtained from the same image acquisition. Since the characteristics of tap A and B are not the same, additional image sets with a delay of half period $\left(I_{3}\right.$ in tap A, $I_{1}$ in tap B) and a delay of three quarter period $\left(I_{4}\right.$ in tap A, $I_{2}$ in tap B) are acquired for a tap correction. Finally, each gated image is obtained by a sum of tap images, e.g. $I_{1}=I_{1, \text { tapA }}+I_{1, \text { tapB }}$.
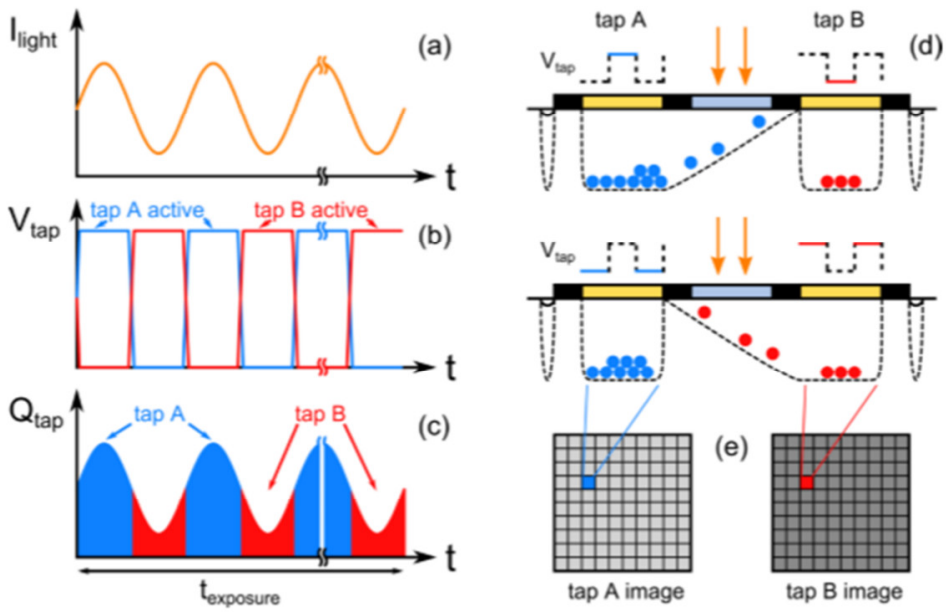

Figure 3. The sinusoidally modulated waveform of light incident on one pixel of the image sensor over time is shown in (a). The charge carriers (electrons) are generated by absorption of light in the photodiode and directed into one of two charge buckets, called tap A and B (d). The taps are alternately selected by applying a voltage waveform shown in (b). The rectangular modulation of the tap A control voltage (blue curve) is inverted for the opposite tap B (red curve) [3].

\section{Modulation light source}

It is important for the FLIM-PSP technique to use a reliable and stable sinusoidal excitation light [4]. In this work, the excitation light intensity with sinusoidal modulation is generated by a HARDsoft UV-LED [5], which was modified for our purpose. Figure 4 shows an oscillogram of input signals to the LED and an emitted light from LED. This LED can be operated with a sinusoidal input signal of $1 \mathrm{Vpp}$ generated by the pco.flim camera and a gate input (TTL signal from pco.flim) for switching the generated light on and off. Detailed features of the modulation light source are introduced in the Ref[5]. 


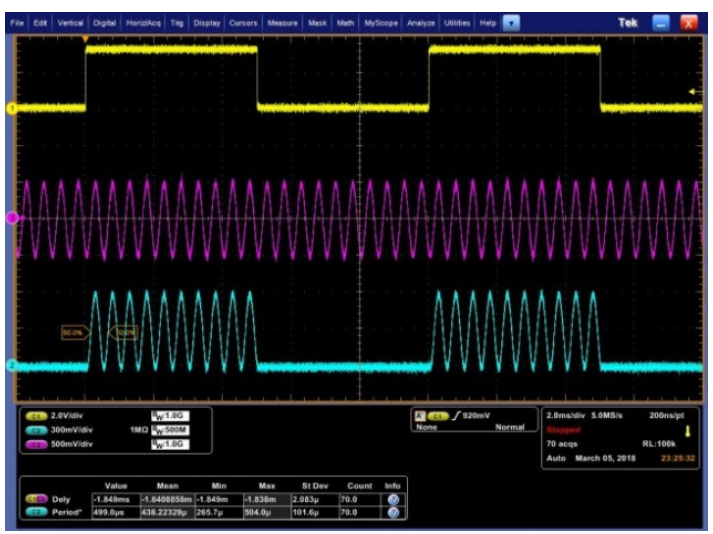

Figure 4. Oscillogram of TTL gate input signal (yellow), sine modulation input signal (magenta) and measured light flux (cyan). LED current $10 \mathrm{~A}$ max and offset $0.53 \mathrm{~V}$ [5].

\section{Calibration Test}

\section{A. PSP}

The paint used in this work is composed of platinum-meso-tetra(pentafluorophenyl)porphine (PtTFPP) [6] as a sensor dye and poly(4-tert-butyl styrene) as a polymer binder. This paint has approximately a lifetime of $10 \mu \mathrm{s}$ at ambient conditions and $50 \mu \mathrm{s}$ at vaccum condition [7,8]. Before appling this PSP coating on a calibration test piece, two additional paint layers, a white polyurethane screen layer and an intermediate layer, are applied. The intermediate layer is composed of white pigments and the polymer used in the PSP coating. This layer works as a barrier coating to prevent an interaction between the PSP coating and the white screen coating.

\section{B. Setup and Conditions}

A bandpass filter around $385 \pm 70 \mathrm{~nm}$ was attached in front of LED and a bandpass filter around $650 \pm 50 \mathrm{~nm}$ was attached to the camera to separate the PSP emission light. A modulation frequency of the sinusoidal excitation light was set to $20 \mathrm{kHz}$ consequently the image gate width was $25 \mu \mathrm{s}$. The LED current was set to $10 \mathrm{~A}$ max with $5 \mathrm{~A}$ offset. A total exposure time of each gated image was set to $110 \mathrm{~ms} .32$ images were acquired and averaged for each gate to enhance the signal-to-noise ratio of the PSP data.

The calibration test was performed at a pressure range between $P=5 \mathrm{kPa}$ and $100 \mathrm{kPa}$ and the temperature range between $T=293 \mathrm{~K}$ and $313 \mathrm{~K}$ (5 K step).

\section{Results}

Figure 5 shows the pressure and temperature dependency of tangent $\Phi$ and $m_{e m}$. For a simpler post image processing tangent $\Phi$ is used instead of $\Phi$. It is shown that both parameters have sensitivity to pressure and temperature.

In order to correct for inhomogeneous lifetime distribution over the model surface each parameter is normalized by the reference condition [7,9]. Figure 6 shows the normalized plot of each parameter by the reference condition at $P_{\text {ref }}=60 \mathrm{kPa}$ and $T_{r e f}=303 \mathrm{~K}$. Figure 7 shows pressure sensitivities of both parameters at $T=303 \mathrm{~K}$. The phase angle shows higher sensitivity compared to the demodulation index, especially at higher pressure range. The pressure sensitivity of the phase angle is comparable to the conventional two-gated lifetime technique [8]. For the pressure calculation in the wind tunnel test, a 4th-order polynomial equation was used to fit the calibration data. 


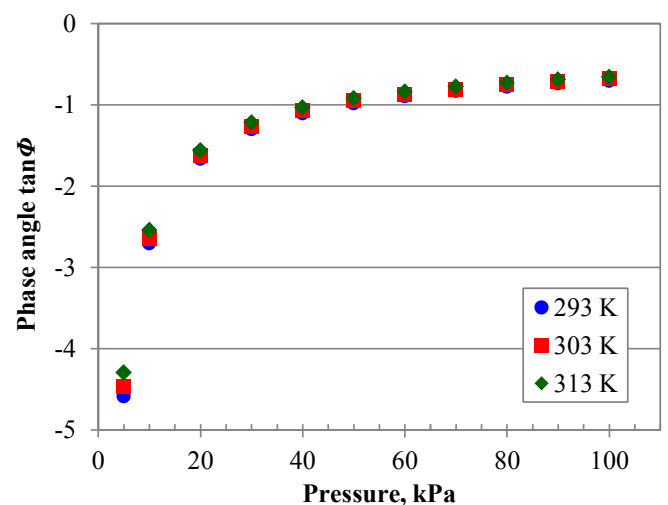

(a) Phase angle (tangential), $\tan \Phi$

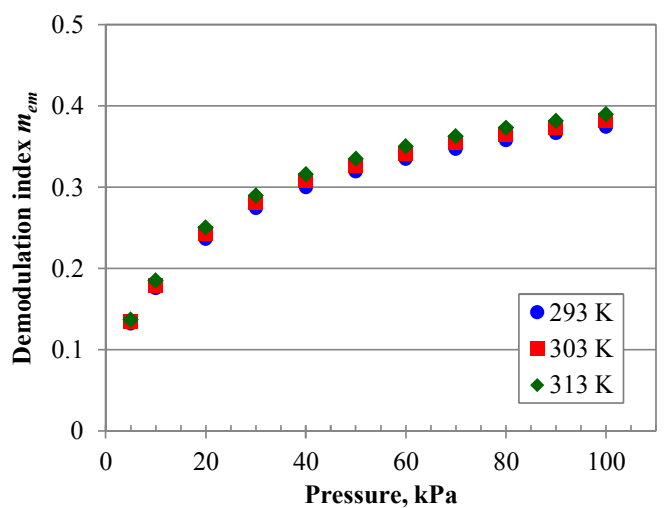

(b) Demodulation index, $m_{e m}$

Figure 5. Pressure and temperature dependency of each parameter.

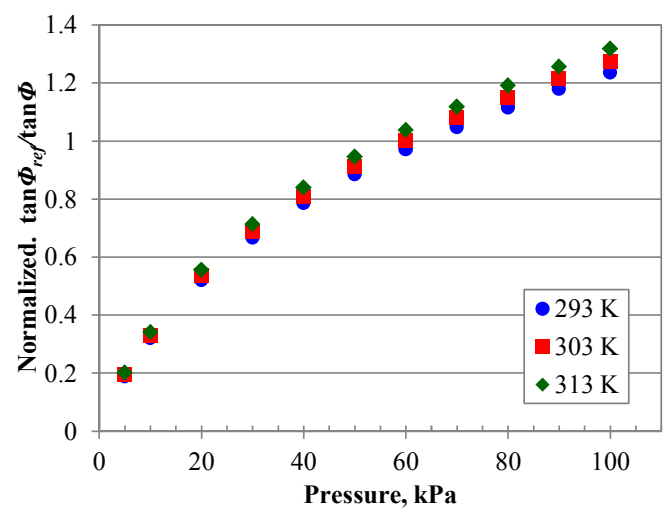

(a) Normalized phase angle (tangential), $\tan \Phi_{\text {ref }} / \tan \Phi$

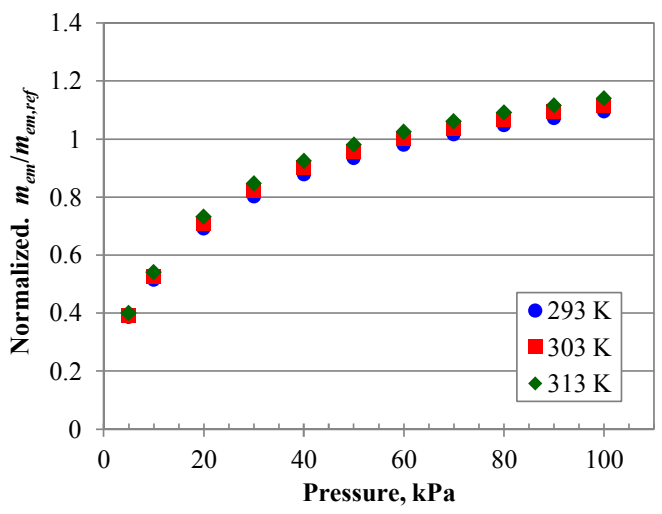

(b) Normalized demodulation index, $m_{e m} / m_{e m, r e f}$

Figure 6. Pressure and temperature dependency of each normalized parameter.

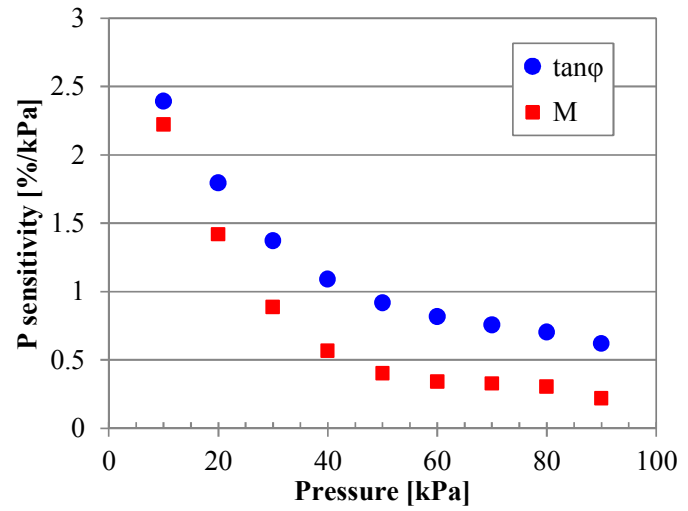

Figure 7. Pressure sensitivities at $T=303 \mathrm{~K}$. 


\section{Wind Tunnel Test}

\section{A. Setup}

Evaluation tests of the FLIM-PSP technique were conducted in the Transonic Wind Tunnel in Göttingen (TWG). The test section size is $1 \mathrm{~m} \mathrm{x} 1 \mathrm{~m}$ and the operation range of the wind tunnel is from $M=0.3$ to 2.2.

As a test model a delta-wing model was used [10]. The same PSP coating as well as camera and LED operation parameters were used in the calibration and wind tunnel tests. The coating was applied on the suction side of the model wing and body part.

The pco.flim camera and the UV-LED were mounted on top of the test section. The distance between the model and equipment was approximately $600 \mathrm{~mm}$. The forward part of the model was not included in the PSP images because of a limited view angle of the setup.

The reference image of PSP was acquired at wind-off condition. The tangent of phase angle and the demodulation index were calculated from Eqs. 1 and 2 after image ensemble averaging and dark image subtraction. Finally, the pressure was calculated from the ratio of the reference and wind-on conditions using the calibration test results.

\section{B. Results}

One data point measured by the FLIM-PSP technique is introduced in Fig. 8. These pressure distributions were calculated for the flow Mach number of 0.85 and high model angle-of-attack. Pressure patterns caused by leadingedge vortices are successfully visualized by both phase angle $\Phi$ and demodulation index $m_{e m}$.

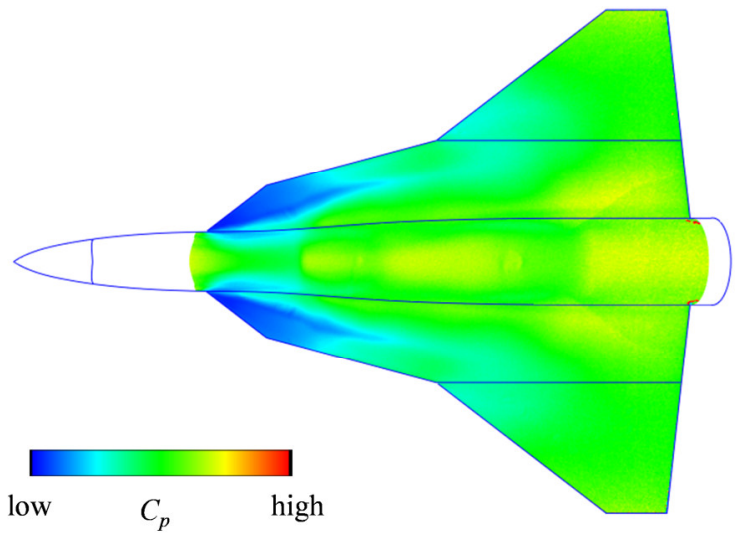

(a) from phase angle (tangential)

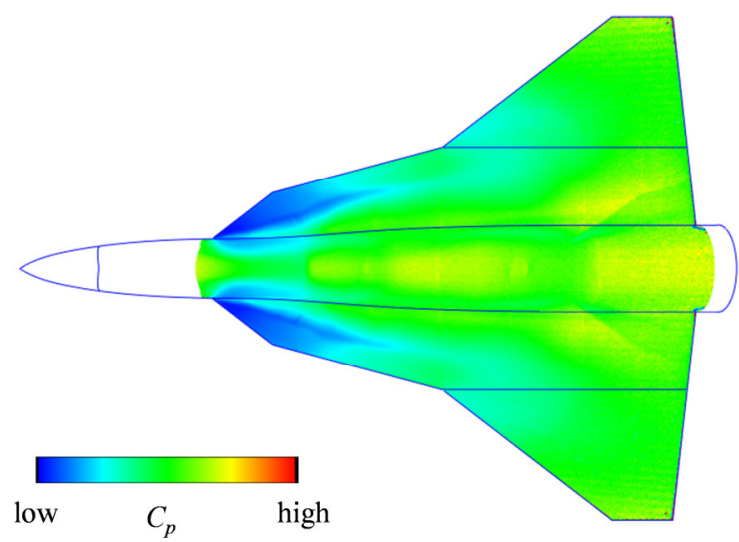

(b) from demodulation index

Figure 8. Pressure distributions obtained by the FLIM-PSP technique.

\section{Measurement Accuracy and Errors}

In this test, four pressure taps, two near the leading edge and two near the wing tip, are available on the area coated with PSP (Fig. 9). The comparison of the pressure tap data and PSP data is summarized in Table 1. Both PSP results from the phase angle and modulation index match well with the pressure tap data. In this test, PSP data at the tap locations P1 and P2 shows consistently higher pressure values compared to the pressure taps. This could be a result of paint aging which over time leads to changing local pressure sensitivities and lifetime patterns appearing at wind-off conditions, as shown in Fig. 10 where the footprints of the leading-edge vortices appear as different lifetime values in the wind-off image. The aging is a result of prolonged preliminary tests on the same coating and in order to correct for these effects the correction of the calibration data is required.

Figure 11 shows effects of the image averaging to the signal-to-noise ratio (SNR) of PSP data. The SNRs are calculated from an area of 50x50 pixels at a mid-part of the wing. The PSP image without spatial filtering is used. The SNR of the demodulation index is nearly two times higher than that of the phase angle. However, as shown in Fig. 7, the pressure sensitivity of the demodulation index is only half than that of the phase angle. Therefore, the final PSP data converted to pressures shows comparable SNR level between the phase angle and the demodulation index. It is noted that the SNR of the conventional lifetime technique used in DLR (two-gate method with CCD camera and 
pulsed LED) with 8 image averaging [8] is approximately three times higher compared to the current FLIM-PSP technique with 32 image averaging.

In Fig. 11, fitted curves with a square root function are also plotted. The increase of SNR with the number of averaging fits very well to the square root function in the phase angle but not in the demodulation index. This indicates that the dominant noise component in the phase angle is the shot-noise and the modulation index has an additional noise component. Figure 12 shows enlarged PSP images near the wing tip. The colormaps are adjusted to the same pressure range in both images. The shot-noise in these results is further reduced by a spatial filtering. It is visible that the PSP result in demodulation index image contains stripe patterns which are caused by a camera-fixed noise. This camera-fixed noise seems to be a constant component in each camera pixel. Therefore, the subtraction of two different gated images (e.g. $I_{1}-I_{3}$ ) can remove this noise. Based on Eqs. 1 and 2, the phase angle is free from this type of noise, however, for the demodulation index the noise remains in the denominator part. A proper camera flat-field correction would be required for removing the camera-fixed noise from the demodulation index results.

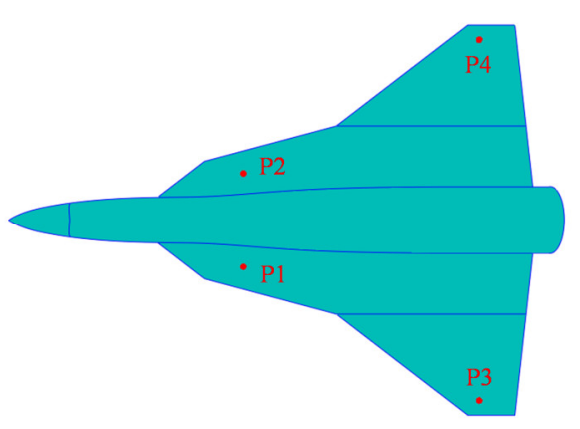

Figure 9. Pressure tap locations.
Table 1 Comparison of the pressure tap data and PSP data

\begin{tabular}{ccc}
\hline & \multicolumn{2}{c}{ Pressure difference in $C_{p}\left(C_{p, p s p}-C_{p, \text { tap }}\right)$} \\
Tap name & Phase angle & Demodulation index \\
\hline P1 & +0.067 & +0.047 \\
P2 & +0.069 & +0.044 \\
P3 & -0.001 & +0.010 \\
P4 & -0.018 & -0.077 \\
\hline RMS & 0.049 & 0.050
\end{tabular}

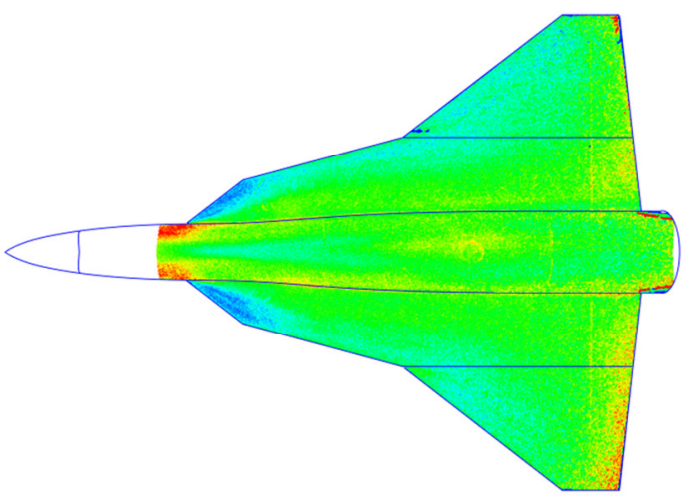

Figure 10. Inhomogeneous lifetime distribution under wind-off condition (phase angle). 


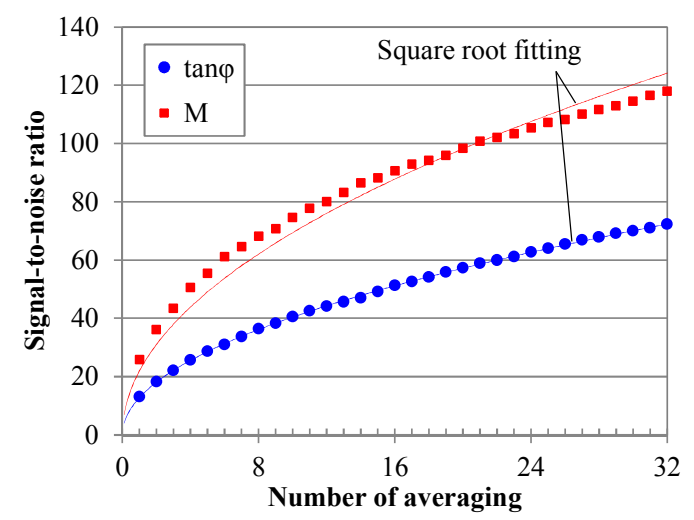

Figure 11. Number of image averaging vs SNR.

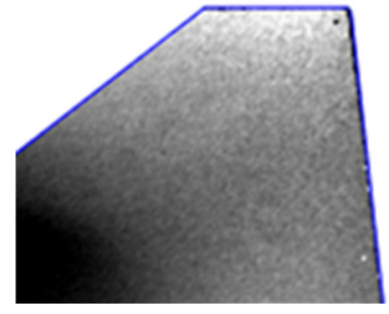

(a) Phase angle

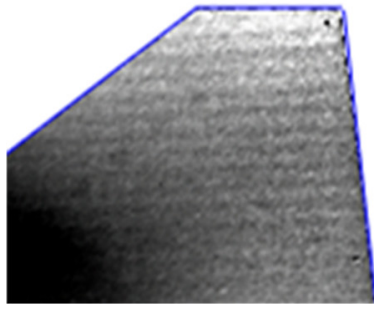

(b) Demodulation index
Figure 12. Enlarged PSP images near the wing tip.

\section{Future Works}

Further investigation for the optimization of measurement parameters is required; e.g. the modulation light frequency, the ratio of the amplitude and the averaged power of the excitation light. The camera calibration is also important to obtain higher quality PSP images by pco.flim camera.

One great interest of the FLIM-PSP technique is a simultaneous pressure and temperature measurement based on the different sensitivity to pressure and temperature in the phase angle and the demodulation index. DLR is starting the investigations of this topic in laboratory tests.

\section{Conclusions}

The applicability of a frequency-domain fluorescence lifetime imaging (FLIM) with a new CMOS camera sensor (pco.flim) to Pressure-Sensitive Paint (PSP) measurement in wind tunnel tests was investigated in calibration tests and in transonic wind tunnel test. In the calibration tests, two lifetime parameters obtained in the FLIM-PSP technique, the phase angle and demodulation index, showed different sensitivity to pressure and temperature. This indicates a potential of simultaneous pressure and temperature measurement. In the wind tunnel test, the FLIM-PSP technique was demonstrated using a delta-wing model. The obtained PSP results successfully visualized the complex pressure distribution on the model surface and the quantitative agreement with conventional pressure tap data was good.

\section{Acknowledgement}

The authors would like to thank the Dr. B. Stasicki in German Aerospace Center and HARDsoft Microprocessor Systems for the development of the LED unit for FLIM-PSP technique. We would also like to thank Dr. R. Franke in PCO AG for his kind supports to the pco.flim camera operation. This work was supported by cooperation project Diabolo jointed run by Airbus Defence and Space GmbH and DLR.

\section{References}

[1] Liu. T. and Sullivan J. P., "Pressure and Temperature Sensitive Paints", Springer-Verlag, Berlin, 2005.

[2] Holmes. J. W., "Analysis of radiometric, lifetime and fluorescent lifetime imaging for pressure sensitive paint", Aeronautical Journal -New Series- 102(1014):189-194, 1998.

[3] Franke. R. and Holst G. A., "Frequency-domain fluorescence lifetime imaging system(pco.flim) based on an in-pixel dual tap control CMOS image sensor", Proc. of SPIE, Vol.9328, 93281K, 2015.

[4] Munekata, M., Yorita, D., Hene, U., Klein, K. and Holst, G. A., "Investigation of Frequency-domain Lifetime PSP Technique using a Fluorescence Lifetime Imaging (FLIM) Camera", 18th International Symposyum on Flow Visualization, Zurich, Switzerland, $26-29$ June 2018.

[5] Stasicki, B., Henne, U., Klein, C., Ludwikowski, K., Munekata, M., Yorita, D. and Holst, G. A., "A Sine-Modulated High-Intensity UV-LED Light Source for Pressure-Sensitive Paint (PSP) Applications using Florescence Lifetime Imaging (FLIM) Technique", The 32th International Congress on High-speed Imaging and Photonics, Twente, Netherland, 9-12 Octber, 2018.

[6] Puklin, E., Carlson, B., Gouin, S., Costin, C., Green, E., Ponomarev, S., Tanji, H. and Goutermann M., "Ideality of Pressure-Sensitive Paint. I. Pl atinum tetra(pentafluorophenyl)porphine in fluo- reacrylic polymer”, Journal of Applied Polymer Science, Vol.77, Issue 13, Page 2795-2804, July 2000. 
[7] Yorita, D., Klein, C., Henne, U., Ondrus, V., Beifuss, U. and Bruse, M., "Improvement of PtTFPP-based PSP for Lifetime-based Measurement and Application to Transonic Wind Tunnel Test", 16th International Symposium on Flow Visualization - ISFV16, Okinawa, Japan, 24-28 June, 2014.

[8] Yorita, D., Henne, U., and Klein, C., "Improvement of Lifetime-based PSP Technique for Industrial Wind Tunnel Tests", AIAA 2017-0703.

[9] Ruyten, W., Sellers, M. E. and Baker, W. M., "Spatially Nonuniform Self-Quenching of the Pressure-Sensitive Paint PtTFPP/FIB", AIAA 2009-1660.

[10]Henne, U., Yorita, D. and Klein, K., "Experimental Aerodynamic High Speed Investigations using Pressure Sensitive Paint for Generic Delta Wing Planforms", 21. DGLR-Fachsymposium der STAB, Darmstadt, Germany 6-7 November, 2018. 